be applied to maintain equilibrium is a tension in the plane of the surface. The thermodynamic relations are

$$
\frac{\partial \mu}{\partial \gamma T n}=-\bar{A} \text { and } \frac{\partial \mu}{\partial T_{\gamma n}}=-\bar{s}, \quad .
$$

where $\gamma$ is the surface tension, $\bar{A}$ is specific area and $\bar{s}$ is the specific entropy of the surface. The relationship between surface tension and temperature is

$$
\frac{\partial \gamma}{\partial T}=-\frac{L}{T \bar{A}}, \quad . \quad \text {. . }
$$

where $L$ is the latent heat of the liquid-surface transition. The difference in sign of expressions 2 and 4 is due to the fact that tension and pressure must be considered to be of opposite sign. The expressions are similar because the tension stress in the surface of a liquid is there for the same reason as would be the hydrostatic tension on a liquid in equilibrium with its solid, at a temperature below the stress-free melting point. The tensions are necessary to reduce the intrinsic potentials of the surface in the one case, and the liquid in the other, so that they equal the intrinsic potentials of the adjacent phases.

A more detailed picture of how the tension stress reduces the intrinsic potential of surface molecules may be obtained as follows. When a molecule escapes from the surface of a liquid, the neighbouring surface molecules move so as to fill up the hole which would otherwise be left by the escaping molecule. The area of the surface is thus reduced by the effective area of the escaping molecule. If acting in the plane of the surface, there is a constant tension force $(\gamma)$; work must be done against this force which equals $\gamma A$, where $A$ is the effective area of a molecule. At the same time, the strain energy associated with the molecule is lost. This amounts to $\int_{A_{0}}^{A} \gamma d A$, where $A$ and $A_{0}$ are the effective areas occupied by a molecule when the surface is under tension $\gamma$ and zero force respectively. The net work done by the tension in the plane of the surface when a molecule escapes from the surface is therefore

$$
-\gamma A+\int_{A_{0}}^{A} \gamma d A=-\int_{0}^{\gamma} A d \gamma . \quad . \quad .
$$

The right-hand side of this expression when differentiated and computed per unit mass instead of per molecule is equal to $-\bar{A}$, which by the first of equations (3) is equal to $\partial \mu / \partial \gamma$. The tension in the surface, therefore, reduces the intrinsic potential of the surface, because of the work done against the tension when a molecule escapes from the surface.

For substances in which the range of molecular action exceeds the effective dimensions of a molecule, the intrinsic potential of molecules near, but not actually in, the surface would exceed that of interior molecules, and some tension force (although it will be much smaller than that necessary between the outermost molecules) will be necessary to reduce their $\mu$ to that of interior molecules. The surface tension must thus be regarded as acting over a region a few molecules thick, the intensity of the force decreasing rapidly with increasing distance from the surface.

We are now in a position to discuss how the tension is set up and maintained. Imagine a stress-free surface of given area. The $\mu$ of surface molecules is higher than that of interior molecules. More mole- cules, therefore, leave the surface than enter it, so that fewer molecules are left to occupy the given area of surface. Their spacing in the plane of the surface therefore exceeds the stress-free spacing, and a tension stress is set up between them. Molecules continue to leave the surface until the tension stress is increased to that necessary to equalize the intrinsic potentials of surface and interior molecules. At this stress, equilibrium is attained, and just as many molecules enter the surface as leave it. The equilibrium tension is then automatically maintained. There is no need to postulate any special orientation of surface molecules or to try to show that there is no shear stress associated with the tension. The tension stress in the surface is continually trying to relax, but as fast as it does so the $\mu$ of surface molecules is increased, and then more molecules leave the surface than enter it until the surface is tightened up. If for any reason the tension became too high, the intrinsic potential of the surface would be too low, and more molecules would enter the surface than leave it, until the tension in the surface was slackened off. This is a fascinating process. In the plane of the surface of a liquid so mobile that millions of molecules per square centimetre are entering and leaving the surface per second, there exists a tension force which cannot relax.

' Bakker, G., "Wien-Harms Handb. Exptphys.”, 6 (1928).

${ }^{2}$ Brown, R. C., Proc. Phys. Soc., in the press.

${ }^{3}$ Adam, N. K., "The Physics and Chemistry of Surfaces" (Oxford Univ. Press, 1941), 5 .

"Rice, J., "Commentary on the Scientifle Writing of J. W. Gibbs" (Yale Univ. Press), 1, 505

\section{INTRODUCTION OF NEW ELECTRICAL AND LIGHT UNITS}

GIR CHARLES DARWIN, director of the National $\checkmark$ Physical Laboratory, has issued the following announcements with regard to the introduction of new electrical and light units, in accordance with the decisions taken by the International Committee of Weights and Measures at its meeting in Paris in October 1946.

\section{Electrical Units}

The system of electrical units employed at present at the National Physical Laboratory are the 'international' units, which are based on certain material standards. From January 1, 1948, the units employed will be those derived from the centimetre, gram and second, that is, the so-called 'absolute' units. The effects of this change may be seen from the following table :

$$
\begin{array}{rlrlrl}
1 \text { international ohm } & =1.00049 & \text { 'absolute' ohms } \\
\text { volt } & =1.00034 & & \text { volts } \\
, & \text { ampere } & =0.99985 & & \text { ampere } \\
\text { watt } & =1.00019 & & \text { watts } \\
, & \text { henry } & =1.00049 & & \text { henries } \\
\text { farad } & =0.99951 & & \text { farad }
\end{array}
$$

The factors given in this table apply to the mean international units; that is to say, to the mean values of the international units as realized at the various national laboratories.

After January 1, 1948, apparatus submitted to the Laboratory with the view of the issue of a National Physical Laboratory Certificate of Examination will be regarded as acceptable if its errors fall within the specified limits when the values are expressed in either international or absolute units, provided that it was 
manufactured before January 1, 1949, and is marked accordingly. Apparatus manufactured after that date, and any apparatus not marked with the date of manufacture, will be acceptable only if its errors fall within the specified limits when the values are expressed in absolute units.

\section{Unit of Light}

The National Physical Laboratory will, on and after January 1, 1948, express all photometric values in terms of units based on the 'new candle'. This unit of luminous intensity is of such a magnitude that the brightness of a full (or cavity) radiator (black body) at the temperature of solidification of platinum is 60 'new candles' per square centimetre. The 'new lumen' is the luminous flux radiated within unit solid angle by a uniform source having a luminous intensity of one 'new candle'. The new unit of illumination will be one 'new lumen' per unit area. One 'new lumen' per sq. ft. will also be called one 'new footcandle', and one 'now lumen' per sq. metre one 'new lux'. The new unit of brightness will be one 'new candle' per unit area, and, alternatively, one 'new foot-lambert'. The latter unit is defined as the brightness of a perfectly diffusing surface of 100 per cent reflexion factor when its illumination is one 'new lumen' per square foot.

When differences of colour are involved in the determination of any magnitude in terms of the above units, the evaluation will be in accordance with that which would be obtained by an observer having the relative luminosity curve (the curve connecting eye sensitivity with wave-length) adopted by the International Commission on Illumination in 1924 and later by the International Committee of Weights and Measures.

The differences between the new units and those in use hitherto are small. The present units, based. on the 'international candle', were introduced on April 1, 1909 , in Great Britain, France and the United States of America, and were adopted in 1921 by the International Commission on Illumination. Nevertheless, Germany and some Central European countries continued to use units based on the Hefner candle (about 0.9 international candle). The international candle was not based on any primary standard; it was derived from the units defined in terms of the old flame standards, and was maintained by means of carbon and tungsten filament electric lamps. Further, the values of the unit at different colour temperatures did not exactly agree, when compared on the basis of the international relative luminosity curve adopted in 1924.

The magnitude of the new unit has been so chosen that it will introduce only very small changes (less than 0.5 per cent) in the values of luminous intensity assigned to lamps operating at a colour temperature of about $2,360^{\circ} \mathrm{K}$. For lamps at considerably higher colour temperatures, for example, ordinary gas-filled electric lamps at normal efficiencies, the values expressed in terms of the 'new candle' will be several per cent lower than those expressed in international candles. At the colour temperature of the primary standard $\left(2,046^{\circ} \mathrm{K}\right.$.), values in terms of the 'new candle' are about $1 \cdot 7$ per cent higher than those in terms of the international candle.

On account of this alteration in the basis on which sources of light of different colours are compared, it is impossible to give a factor for converting values in international candles to values in 'new candles'.

\section{U.S. NATIONAL ACADEMY OF SCIENCES}

\author{
Elections and Awards
}

A $\mathrm{T}$ the annual meeting of the U.S. National. Academy of Sciences held in Washington during April 28-30, the following officers, members, and foreign associates were elected :

President (July 1, 1947-June 30, 1951) : Alfred N. Richards, vice-president in charge of medical affairs, University of Pennsylvania (in succession to Dr. Frank B. Jewett).

Home Secretary (re-elected for a further term of four years ending June 30, 1951): F. E. Wright, Carnegie Institution of Washington, Washington, D.C.

Members of the Council of the Academy (for a threeyear term onding June 30, 1950): W. Albert Noyes, jun., chairman, Department of Chemistry, University of Rochester, Rochester, N.Y.; and Donald D. Van Slyke, chief chemist, Hospital of the Rockefeller Institute for Medical Research, Now York City.

Members : Luis W. Alvarez, professor of physics, University of California, Berkeley, California ; Robert F. Bacher, U.S. Atomic Energy Commission, Washingtion, D.C. ; Paul D. Bartlett, professor of chemistry, Harvard University, Cambridge, Mass.; Jacob Bjerknes, professor of physics, University of California at Los Angeles, California; Francis G. Blake, dean of Yale University School of Medicine, Now Haven, Conn.; R. Alexander Brink, chairman of the Department of Genetics, University of Wisconsin, Madison, Wisconsin ; Ralph W. Chaney, professor of paleobotany and curator of paleobotany, Museum of Paleobotany, University of California, Berkeley, California; Arthur C. Cope, professor in charge of the Department of Chemistry, Massachusetts Institute of Technology, Cambridge, Massachusetts ; Farrington Daniels, professor of physical chemistry, University of Wisconsin, Madison, Wisconsin ; Arnold Gesell, director, Clinic of Child Development, Yale University School of Medicine, New Haven, Connecticut; James Gilluly, professor of geology, University of California at Los Angeles, California; R. B. Goldschmidt, professor of zoology, University of California, Berkeley, California; Samuel A. Goudsmit, professor of physics, Northwestern University, Evanston, Illinois; C. H. Herty, jun., research engineer and assistant to vice-president, Bethlehem Steel Company, Bethlehem, Pennsylvania ; Frederick L. Hisaw, professor of zoology, Harvard University, Cambridge, Massachusetts; Wolfgang Köhler, professor of psychology, Swarthmore College, Pennsylvania; L. G. Longsworth, associate member, Rockefeller Institute for Medical Research, New York City ; Edwin M. McMillan, professor of physics, University of California, Berkeley, California ; Walter J. Meek, acting dean, Medical School, University of Wisconsin, Madison, Wisconsin; J. L. Oncley, director, Ultracentrifuge Laboratory, Harvard University Medical School, Boston, Massachusetts; Lars Onsager, professor of chemistry, Yale University, New Haven, Conn.; John P. Peters, professor of medicine, Yale University, New Haven, Conn.; Paul A. Smith, professor of mathematics, Columbia University, New York City ; C. Richard Soderberg, professor of applied mechanics, Massachusetts Institute of Technology, Cambridge, Massachusetts ; Paul Weiss, professor of zoology, University of 